\title{
High Resolution Radio Continuum Observations Of High Mass Star Formation Regions
}

\author{
S. Kurtz \\ Universidad Nacional Autónoma de México Apdo. Postal 3-72 Xangari, \\ C.P. 58089 Morelia, Mich. Mexico \\ P. Hofner, C. Vargas, \& W. Díaz-Merced \\ University of Puerto Rico, Río Piedras P.O. Box 23343, Rio Piedras \\ PR 00931-3343 USA
}

\begin{abstract}
We present high angular resolution centimeter and millimeter continuum observations of several galactic massive star formation regions. Using calibration techniques pioneered at the Very Large Array, we are able to obtain high quality images even under adverse conditions of phase stability. Techniques such as these will be essential if future millimeter arrays are to obtain high quality and high precision images. We provide a current summary of our on-going survey, and present images and a brief discussion of several of the more intriguing sources.
\end{abstract}

\section{Introduction, Observations and Preliminary Results}

We present preliminary results of our Very Large Array (VLA) survey in the masing lines of $\mathrm{CH}_{3} \mathrm{OH}$ at $23.121 \mathrm{GHz}$ and $44.069 \mathrm{GHz}$. The chief goal is to provide accurate positions for the methanol masers. Accurate maser positions will allow us to: 1) determine in which astrophysical environment methanol masers occur, 2) test the classification scheme and inversion mechanisms proposed for $\mathrm{CH}_{3} \mathrm{OH}$ masers, and 3) provide a catalog of strong, pointlike sources for phase calibration at $7 \mathrm{~mm}$ for observations of high-mass star formation regions.

Using the $\mathrm{C}$ and $\mathrm{D}$ configurations of the VLA, we simultaneously observed the $\mathrm{CH}_{3} \mathrm{OH} 7_{0}-6_{1} \mathrm{~A}^{+}$transition at $44.069 \mathrm{GHz}$ and the $\mathrm{CH}_{3} \mathrm{OH} 9_{2}-10_{1} \mathrm{~A}^{+}$ transition at $23.121 \mathrm{GHz}$. The sensitivity of these observations was better than $1 \mathrm{Jy}$, and the velocity coverage was $22 \mathrm{~km} \mathrm{~s}^{-1}$ at $44 \mathrm{GHz}$ and $41 \mathrm{~km} \mathrm{~s}^{-1}$ at $23 \mathrm{GHz}$. The positional precision of the resulting maser detections is better than one second of arc. About 30 regions were observed in these two runs; an additional dozen or so sources will be observed in a third and final observing run in September 2000 in the D array.

At present we have mapped 15 sources in the $23 \mathrm{GHz}$ (class II) line and 6 sources in the $44 \mathrm{GHz}$ (class I) line. Most of the $44 \mathrm{GHz}$ masers are not coincident with the HII regions in the field and often occur in linear structures. This morphology suggests that these masers may arise in shocked molecular gas due to the interaction of outflows or jets with dense ambient gas. This is exemplified in the case of IRAS 20126+4104, shown in Figure 2. 


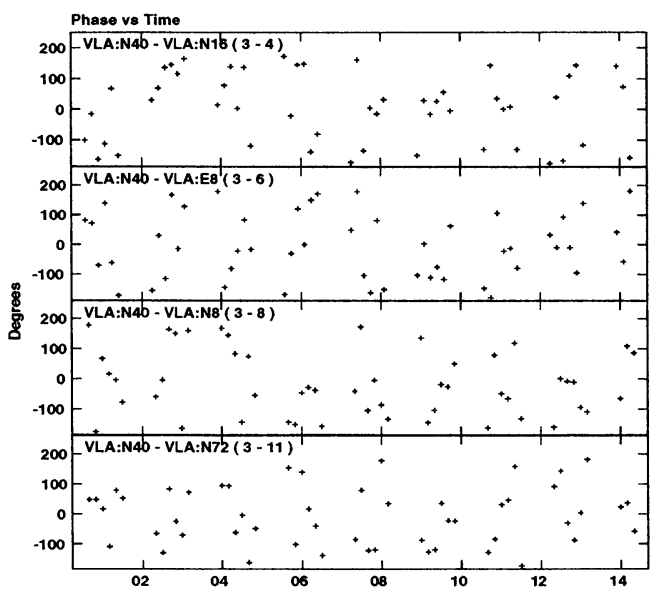

Figure 1. The phases of these data, taken during evening observations in the most recent A configuration, show an all too typical situation for the more distant antennas. The phase variation is too rapid to track, even using fast-switching. Under these conditions, phase referencing with methanol masers offers the only hope of producing images. The continuous observation of the maser allows calibration on timescales as short as a few seconds.

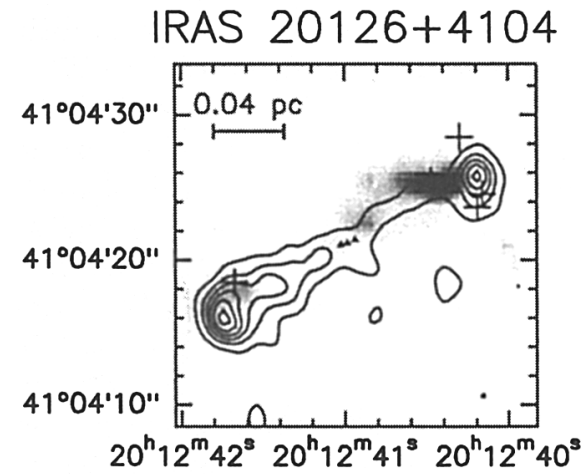

Figure 2. The massive star forming region $20126+4104$ is shown here in shock-excited molecular hydrogen (gray scale) and $\mathrm{SiO}$ (contours). The small triangles in the center of the figure denote water masers, while the crosses show the methanol masers we detected as a part of this survey. That the methanol masers are nearly coincident with shocked gas is strong evidence that these masers arise in outflow material, not in circumstellar disks. 